\title{
Flower and leaf recognition for plant identification using convolutional neural network
}

\author{
Nurul FatihahSahidan, Ahmad Khairi Juha, Norasiah Mohammad, Zaidah Ibrahim \\ Faculty of Computer and Mathematical Sciences, Universiti Teknologi MARA, Malaysia
}

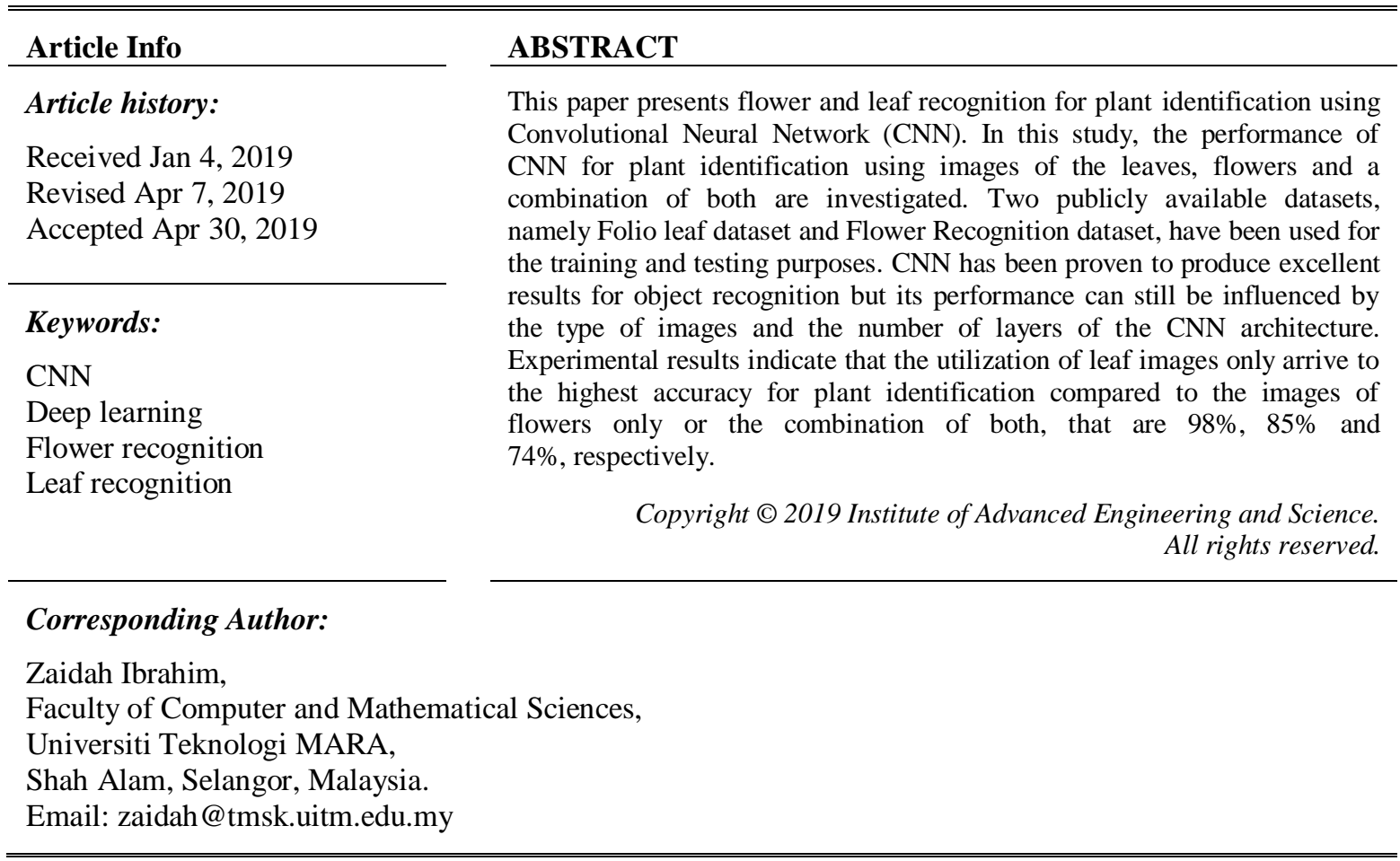

\section{INTRODUCTION}

Deep learning is a machine learning technique that teaches computers to do what comes naturally to humans: learn by example [1]. In deep learning, a computer model learns to perform classification tasks directly from images, text, or sound. Within the past few years, deep learning algorithms particularly Convolutional Neural Networks (CNNs) have proven their much powerful feature representation capabilities in computer vision [2]. Data are trained by using a large set of labeled data with various numbers of layers of the CNN.

Current advances in hardware technology have enabled the evolution of CNN and massive number of their applications, as well as complicated tasks like objects recognition and image classification [3]. It has resulted in ground breaking decisions over the last decade in various fields related to pattern recognition; from image processing to voice recognition [4]. CNN's capabilities have become a known and used in various object recognition problems such as flower categorization [5], leaf recognition [6], voice analysis [7], image classification [8], fruit classification and ripeness grading recognition [9], food recognition [10], and plant disease identification [11].

CNN is a sub-class of Artificial Neural Network (ANN), an information processing paradigm that is inspired by the way the biological nervous system works, that is how the the brain process information. The brain consists of a large number of neurons interacting together to solve certain problems [3, 12]. ANN is a math representation of the human nervous architecture that belongs to artificial intelligence [13]. It is still unclear whether the ANN can modify and adapt itself to explain and adapt itself to overcome various other tasks as opposed to CNN that has proven to be effective in the field of major tasks [14].

As in other image recognition tasks, plant identification depends on computational strategies to extract discriminative options from pictures. Options are historically hand-loomed or hand designed. 
However, a recent trend in machine learning has shown that learned representations are more practical and economical [15]. Several parts of a plant can be used by a botanist in order to recognize a plant and various efforts have been done that includes flowers, leaves, and roots [16]. Leaves are the most widely used as it is more convenient to be examined. Besides that, it provides important diagnostic characters and discriminative features for plant identification and the results are great [17-19]. However, some researches have also been made on flower recognition to identify the species of plants. Even though flowers have many different species, some of them have very similar characteristics and looks. This similarity and dissimilarity make the flowers recognition process with a highly accurate result is very challenging [20].

The purpose of identifying plants is to categorize the plants for recording purposes. The process of identifying a plant using flowers and leaves are an easy task for botanists as they can simply recognize it using their knowledge [21]. On the contrary, for machines to achieve the same recognition results requires performing image-processing techniques to extract visual information and compare them to existing sets of data [7]. Structured learning or better known as deep learning, has been recognized as a new area in computer vision that has been reported to produce excellent results [22].

Research about flower and leaf recognition has been conducted by several researchers using various techniques. Hu's seven moment algorithm has been applied for flower recognition with almost $80 \%$ accuracy [23]. With data augmentation, the accuracy of $99.04 \%$ using AlexNet and 99.42 using GoogleNet have been obtained [24]. Support Vector Machine (SVM) with texture features has achieved 99\% accuracy [20]. Besides that, shape features and colour histogram with k-nearest neighbour classifiers have been applied with 87.2\% accuracy [6]. Since the results of using Folio leaf dataset and Flower dataset were very positive, these datasets were chosen to be experimented in this research.

In this paper, a comparison of the accuracy performance between a set of flowers, a set of leaves and a combination of a set of flowers and leaves have been conducted in order to analyze the accuracy performance of plant identification. This paper is organized as follows. The nest section discusses about $\mathrm{CNN}$ and the features that have been utilized for plant identification, followed by results analysis of the experiments using different datasets and different number of layers on CNN. The last section concludes this paper with the information of future work.

\section{RESEARCH METHOD}

\subsection{Convolutional Neural Network (CNN)}

CNN consists of four types of layers which are convolution layers, pooling layer, Rectified Linear unit $(\mathrm{ReLu})$ layer and fully connected layers. Convolution layers extract the input of an image by using convolution operation and produce a feature map [19]. Multiple convolutional layers can also be applied for different feature maps. This method is to ensure complete extraction of various features. Next, pooling layer lower the size of the feature maps. This process makes the input robust against noise and distortion [2]. CNN particularly relies on the third layer which is the activation function. CNN may use specific functions such as ReLUs functions to efficiently implement non-liner triggering. All negative pixel values in the feature map are replaced with zero in the ReLu layer [6]. Fully connected layer which is the last layer, total the weightage of previous layers of features to determine the output.

Figure 1 shows the CNN architecture that extracts features by using convolution technique on the input image, resizes the feature map during pooling layer and classifies it in the fully connected layer. The first convolution layer usually extracts the low-level features such as edges while the second convolution layer extracts the high-level features such as the shape.

Explaining research chronological, including research design, research procedure (in the form of algorithms, Pseudocode or other), how to test and data acquisition [6-9]. The description of the course of research should be supported references, so the explanation can be accepted scientifically [4, 10].

Tables and Figures are presented center, as shown in Table 1 and Figure 1 and cited in the manuscript before appeared.

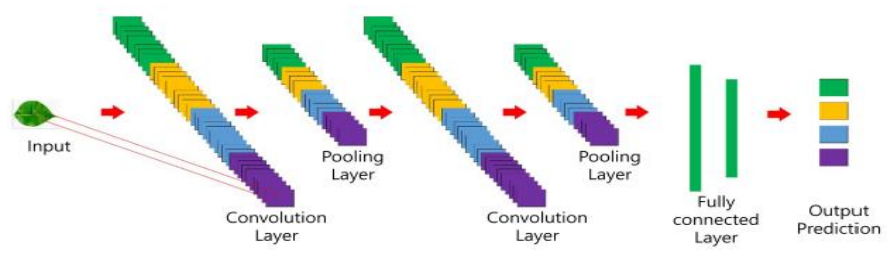

Figure 1. Convolutional neural network architecture [25] 


\subsection{Flower and Leaf Recognition}

When it comes to quantifying flower and leaf images, the three most important attributes to be considered are color, texture and shape.

\subsubsection{Color}

One of the important features to recognize the flower is through the "Color". Color Histogram which calculates the frequency of pixel intensities occurring in an image is the most genuine and simple global feature descriptors [5]. This enables the descriptor to learn about the distribution of each color in an image. The feature vector is taken by combining the count for each color. However, the color features of an image only are not enough to quantify flowers, because in a multi-species environment, two or more species could be of the same color [5]. For example, daisy and magnolia have similar color but they are different flowers, and of course, different plants.

\subsubsection{Texture}

Texture is another important feature that can be used to recognize the species of plants. The Gray Level Co - occurrence Matrices GLCM is one of the most important and earliest texture analysis approaches introduced by (Haralick) in 1973 [24]. According to S. Albawi there are 14 statistical features that can be calculated to quantify an image based on texture and this requires a large amount of input size [4]. The resulting feature vector is a 13-dimensional feature vector that ignores the 14th dimension due to high computational time [5].

\subsubsection{Shape}

Another important feature to quantify the image is through the "shape". Zernike moments, introduced by Teague as a shape descriptor, and Hu moments are two widely used global form descriptors in computer vision research that can represent the shape of an object [5]. Moments depend on the statistical expectations of a random variable. There are seven moments, which are called $\mathrm{Hu}$ moments. The combination of these seven moments form 7-dimensional feature vector [5]. But there are flowers that have similar shape such as peonies and hydrangea

\section{RESULTS AND ANALYSIS}

The laptop used to run the CNN for this project was Asus with Windows 10, Intel Core i5 processor, 4.00 GB RAM and the operating system is 64-bit while the software used is Matlab 2018a. For this project, three CNN tests have been undertaken using two datasets. The datasests used for three experiments are Flower Recognition dataset, Folio Leaf dataset and a combination of both datasets.

All of the images were resized to 224 by 224 pixels to ensure the consistency of the data for each experiment. Experiments were conducted by changing the number of layers, the values of the parameters in the convolve layer, pooling layer and the learning rate. The purpose is to determine the best combination of parameters to produce the highest accuracy for plant recognition from all three datasets.

The results of the experiments were recorded in Table 1. By referring to Table 1, we can see that the first column indicates the number of stacks of layers where a stack consists of one convolve layer, one maxpooling layer and one ReLu layer. In column Convolve layer, the first number in the square bracket represents the size of the convolve filter while the second number represents the number of convolve filters. The third layer represents the size of max-pooling filter and the number of stride. The number of epoch and learning rate is shown in column 4. The number of epoch determines the number of repetitions of all the training data while the learning rate is the amount of adjustment that is being made to the weights during the training process. The adjustments of the weights are performed until the error rate is minimal where it represents the learning process of $\mathrm{CNN}$.

\subsection{Flower Recognition Dataset}

Flower Recognition dataset comprises of 4242 floral images where data collection for all the floral images are scraped from google images, flicr data, and yandex images [25]. However, for the purpose of this project, only 100 images have been randomly chosen from 5 categories which are daisy, dandelion, rose, sunflower and tulip. Figure 2 shows some example images from this dataset. 

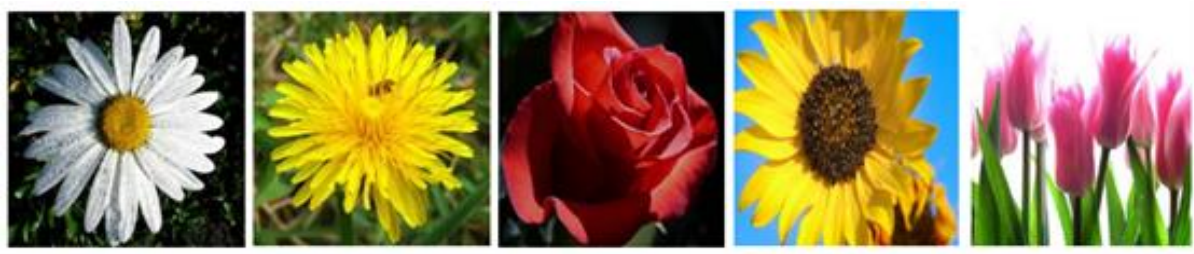

Figure 2. Flower recognition dataset [26]

Table 1 shows the accuracy achieved from CNN testing on Flower Recognition dataset where different stacks of layers have been experimented to examine the effects of the number of layers to the accuracy performance. The highest accuracy was obtained with four stacks of layers which is $74 \%$.

Table 1. Experimental Results on Parameter Tuning for Basic CNN based on Flower Recognition Dataset

\begin{tabular}{|c|c|c|c|c|c|}
\hline $\begin{array}{c}\text { No of Stack } \\
\text { of Layers }\end{array}$ & Convolve Layer & $\begin{array}{l}\text { Pooling } \\
\text { layer and } \\
\text { Stride } \\
\end{array}$ & $\begin{array}{c}\text { Epoch, Learning } \\
\text { Rate }\end{array}$ & Accuracy $(\%)$ & Total Time/s \\
\hline \multirow{2}{*}{1} & {$[3,64]$} & 3 & $10,0.001$ & 68 & $3 \min 18 \mathrm{~s}$ \\
\hline & {$[3,16]$} & 2 & $10,0.001$ & 62 & $3 \min 14 s$ \\
\hline \multirow{2}{*}{2} & {$[3,16],[3,48]$} & 3 & $10,0.001$ & 66 & $6 \min 55 \mathrm{~s}$ \\
\hline & {$[3,64],[3,80]$} & 3 & $10,0.001$ & 70 & $0 \min 34 \mathrm{~s}$ \\
\hline \multirow{2}{*}{3} & {$[3,64],[3,64],[3,128]$} & 3 & $10,0.001$ & 68 & $3 \min 44 s$ \\
\hline & {$[5,20],[3,20],[3,16]$} & 3 & $10,0.001$ & 64 & $3 \min 35 s$ \\
\hline \multirow{2}{*}{4} & {$[3,64],[3,64],[3,80],[3,80]$} & 3 & $10,0.001$ & 64 & $4 \min 35 s$ \\
\hline & {$[3,80],[3,80],[3,256],[3,256]$} & 3 & $10,0.001$ & 74 & $4 \mathrm{~min} 35 \mathrm{~s}$ \\
\hline
\end{tabular}

\subsection{Folio Leaf Dataset}

The second dataset used is Folio Leaf DataSet [26]. Leaves pictures are taken from plants on the farm of the University of Mauritius and nearby locations. There are 32 categories of plant and for each category 20 images of leaves are experimented. However, for this project, only 5 categories and 20 images for each category are used. Figure 3 shows some sample images from this dataset.

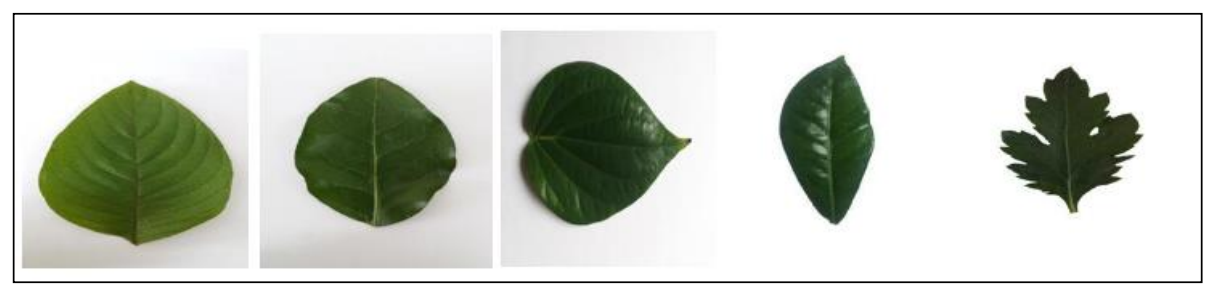

Figure 3. Folio leaf dataset [27]

Table 2 shows the different accuracies achieved from CNN testing on this dataset with different stacks of layers. By looking at Table 2, we can see that the highest accuracy was produced with 4 stacks of layers that is $98 \%$.

Table 2. Experimental Results on Parameter Tuning for Basic CNN based on Folio Leaf Dataset

\begin{tabular}{cccccc}
\hline $\begin{array}{c}\text { No of Stack } \\
\text { of Layers }\end{array}$ & Convolve Layer & $\begin{array}{c}\text { Pooling layer } \\
\text { and Stride }\end{array}$ & $\begin{array}{c}\text { Epoch, } \\
\text { Learning Rate }\end{array}$ & Accuracy $(\%)$ & Total Time/s \\
\hline \multirow{2}{*}{1} & {$[3,16]$} & 3 & $10,0.001$ & 71.92 & $2 \mathrm{~min} \mathrm{32s}$ \\
& {$[5,20]$} & 2 & $10,0.0001$ & 65.62 & $3 \mathrm{~min} 23 \mathrm{~s}$ \\
2 & {$[3,16],[3,16]$} & 3 & $10,0.001$ & 79.81 & $4 \mathrm{~min} 27 \mathrm{~s}$ \\
& {$[3,80],[3,64]$} & 2 & $10,0.001$ & 73.82 & $2 \mathrm{~min} 40 \mathrm{~s}$ \\
3 & {$[3,16],[3,16],[3,32]$} & 3 & $10,0.001$ & 76.66 & $3 \mathrm{~min} 44 \mathrm{~s}$ \\
4 & {$[5,20],[3,20],[3,16]$} & 3 & $10,0.001$ & 82.03 & $3 \mathrm{~min} 35 \mathrm{~s}$ \\
$\mathbf{4}$ & {$[\mathbf{3 , 6 4}],[\mathbf{3 , 6 4}],[\mathbf{3 , 8 0}],[\mathbf{3 , 8 0}]$} & $\mathbf{3}$ & $\mathbf{1 0 , 0 . 0 0 1}$ & $\mathbf{9 8}$ & $\mathbf{3} \mathbf{m i n ~ 2 0 s}$ \\
\hline
\end{tabular}




\subsection{Folio and Leaf Dataset}

In this experiment, a combination of Flower Recognition dataset and Folio Leaf Dataset has been tested. This dataset consists of 10 categories in which 5 categories from the Flower Recognition dataset and 5 categories from the Folio Leaf dataset with a total of 200 images. Table 3 illustrates the accuracies achieved from CNN testing on these datasets where $85 \%$ accuracy has been acquired with four stacks of layers.

Table 3. Experimental Results on Parameter Tuning for basic CNN using Flower Recognition and Folio Datasets

\begin{tabular}{cccccc}
\hline $\begin{array}{c}\text { No of Stack } \\
\text { of Layers }\end{array}$ & Convolve Layer & $\begin{array}{c}\text { Pooling layer } \\
\text { and Stride }\end{array}$ & $\begin{array}{c}\text { Epoch, } \\
\text { Learning Rate }\end{array}$ & $\begin{array}{c}\text { Accuracy } \\
(\%)\end{array}$ & Total Time/s \\
\hline 1 & {$[3,48]$} & 3 & $10,0.001$ & 74 & $5 \mathrm{~min} 15 \mathrm{~s}$ \\
& {$[3,80]$} & 3 & $10,0.001$ & 72 & $5 \mathrm{~min} 12 \mathrm{~s}$ \\
2 & {$[3,64],[3,80]$} & 3 & $10,0.001$ & 76 & $6 \mathrm{~min} 55 \mathrm{~s}$ \\
& {$[3,64],[3,128]$} & 2 & $10,0.001$ & 60 & $7 \mathrm{~min} 25 \mathrm{~s}$ \\
3 & {$[3,64],[3,64],[3,128]$} & 3 & $10,0.001$ & 66 & $9 \mathrm{~min} 56 \mathrm{~s}$ \\
$\mathbf{4}$ & {$[5,20],[3,64],[3,80]$} & 3 & $10,0.001$ & 73 & $3 \mathrm{~min} 46 \mathrm{~s}$ \\
& {$[\mathbf{3 , 6 4}],[\mathbf{3 , 6 4}],[\mathbf{3 , 8 0}],[\mathbf{3 , 8 0}]$} & $\mathbf{3}$ & $\mathbf{1 0 , 0 . 0 0 1}$ & $\mathbf{8 5}$ & $\mathbf{7} \mathbf{m i n} \mathbf{2 6 s}$ \\
\hline
\end{tabular}

\subsection{Overview of All Datasets}

Table 4 provides an overview of the highest accuracy performance produced by CNN conducted on all three datasets. By looking at Table 4, we can see that the best accuracy has been achieved by utilizing Folio Leaf dataset which is $98 \%$. This shows that not all datasets will produce good results when tested with CNN. This has been proven with the Flower Recognition dataset which only achieved $74 \%$ accuracy even when tested using the same specifications with other dataset in which is made to ensure the consistency of this experiment.

Table 4. The Performance Overview for Analysis of CNN for Flower and Leaf Recognition

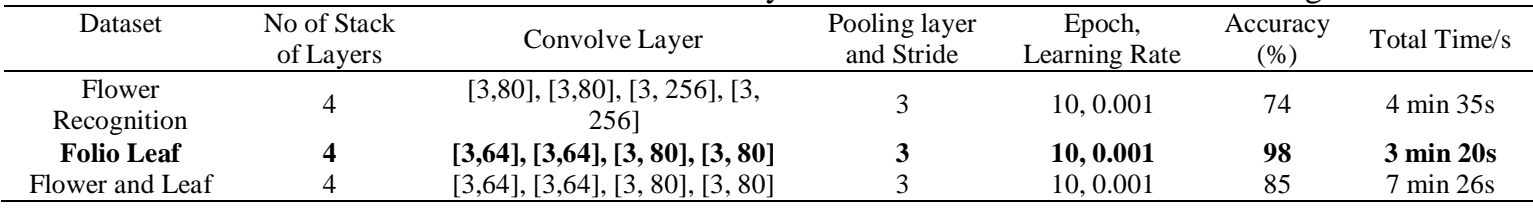

By referring to Table 4, we can see that plant identification based on the images of the leaves are more accurate compared to by using the images of the flowers or a combination of both. The accuracy of flower recognition only reaches $64 \%$ when using the same architecture as stated in Table 4 for leaf recognition and the combination of both

\section{CONCLUSION}

This paper evaluates the performance of CNN for plant identification using the images of the leaves only, flowers only and a combination of both images. The experimental results show that the use of the images of the leaves only arrive to the best accuracy. This may be due to the fact that flowers have too many variations in terms of shapes and colours compared to the leaves. For future research, we plan to investigate other variations of the CNN architecture and compare with other plant datasets for more training data.

\section{ACKNOWLEDGEMENTS}

The authors would like to thank the Institute of Graduate Studies, Universiti Teknologi MARA, and the Faculty of Computer and Mathematical Sciences, Universiti Teknologi MARA, Shah Alam, Selangor, for sponsoring this research.

\section{REFERENCES}

[1] E. Nishani and B. Cico, "Computer Vision Approaches based on Deep Learning and Neural Networks," 2017 6th Mediterranean Conference on Embedded Computing (Meco), Bar, Montenegro, 11-15 JUN 2017.

[2] M. Afiq, et al., "Deep Learning for Roman Handwritten Character Recognition," Indonesian Journal of Electrical Engineering and Computer Science, vol. 12(2), pp. 455-460, Nov 2018.

\footnotetext{
Flower and leaf recognition for plant identification using convolutional neural network (Zaidah Ibrahim)
} 
[3] A. K. Reyes, J. C. Caicedo \& J. E. Camargo., "Fine-tuning Deep Convolutional Neural Networks for Plant Recognition," Laboratory for Advanced Computational Science and Engineering Research, 2015.

[4] S. Albawi, et al., "Understanding of a convolutional neural network," 2017 International Conference on Engineering and Technology (ICET), Antalya, pp. 1-6, 2017.

[5] I, G and V, S, "Flower Species Recognition System using Convolution Neural Networks and Transfer Learning," 2017 4th International Conference on Signal Processing, Communications and Networking, Chennai, Mar 2017.

[6] Munisami. T, et al., "Plant leaf recognition using shape features and colour histogram with k-nearest neighbour classifiers," Procedia Computer Science (Elsevier) Journal, vol. 58, pp. 740-747, 2015.

[7] R. Durratun, et al., "Performance Evaluation for Vision-Based Vehicle Classiffication Using Convolutional Neural Network," International Journal of Engineering \& Technology, vol.7(3.15), pp. 86-90, 2018.

[8] T. Guo, et al., "Simple convolutional neural network on image classification," 2017 IEEE 2nd International Conference on Big Data Analysis (ICBDA), Beijing, pp. 721-724, 2017.

[9] I. Zaidah, et al., "Palm Oil Fresh Fruit Bunch Ripeness Grading Recognition Using Convolutional Neural Network," Journal of Telecommunication, Electronic \& Computer Engineering, vol. 9(3-2), pp. 109-113, 2018.

[10] S. Albawi, et al., "Understanding of a convolutional neural network," 2017 International Conference on Engineering and Technology (ICET), Antalya, pp. 1-6, 2017.

[11] S. Slajodevic, et al., "Deep Neural Networks Based Recognition of Plant Diseases by Leaf Image Classification," Hindawi Publishing Corporation Computational Intelligence and Neuroscience, pp. 11, May 2016.

[12] M. Ghiassi, J. Skinner \& D. Zimbra, "Twitter brand sentiment analysis: A hybrid system using n-gram analysis and dynamic artificial neural network," Procedia Computer Science (Elsevier) Journal. 58, pp. 6266-6282, 2013.

[13] F. Amato, et al., "Artificial neural networks in medical diagnosis," 2013 Journal of Applied Biomedicine, pp. 47-58, Jan 2013.

[14] G. Carlec and M. Troyer, "Solving the Quantum Many-Body Problem with Artificial Neural Networks," 2016 arXiv: 1606.02318v1 [cond-mat.dis-nn], Jun 2016.

[15] S. H. Lee, et al., "How Deep Learning Extracts and Learns Leaf Features for Plant Classification," Pettern Recognition, May 2015.

[16] Y. Sun, et al., "Deep Learning for Plant Identification in Natural Environment," Hindawi Publishing Corporation Computational Intelligence and Neuroscience, pp. 6, May 2017.

[17] A. Fuentes, et al., "A Robust Deep-Learning-Based Detector for Real-Time Tomato Plant Diseases and Pests Recognition," Sensors 2017, 2017.

[18] P. Pawara, et al., "Comparing Local Descriptors and Bags of VisualWords to Deep Convolutional Neural Networks for Plant Recognition," 6th International Conference on Pattern Recognition Applications and Methods (ICPRAM 2017), pp. 479-486, 2017.

[19] I. Zaidah, et al., "Multi-maxpooling Convolutional Neural Network for Medicinal Herb Leaf Recognition," Proceedings of the 6th IIAE International Conference on Intelligent Systems and Image Processing, Shimane, Japan, pp. 327-331, Sep 2018.

[20] Gurnani. A, et al., "Flower Categorization using Deep Convolutional Neural Networks," pp. 4321-4324, 2017.

[21] I. Zaidah, et al., "Leaf Recognition using Texture Features for Herbal Plant Identification," Indonesian Journal of Electrical Engineering and Computer Science (IJEECS), vol. 9(1), pp. 152-156, Jan 2018.

[22] L. Deng and D. Yu, "Deep Learning: Methods and Applications," Foundations and Trends in Signal Processing, vol. 7(3-4), pp. 197-387, 2013.

[23] Tiay T, et al., "Flower Recognition System Based on Image Processing. In Student Project Conference (ICTISPC)," Third ICT International, pp. 99-102, 2014.

[24] M. O. Assayony and S. A. Mahmoud, "Integration of Gabor Features with Bag-of-Features Framework for Arabic Handwritten Word Recognition," 2017 9th IEEE-GCC Conference and Exhibition (GCCCE), Manama, pp. $1-4,2017$.

[25] J.W. Su and R.S. Yong, "Plant Leaf Recognition Using a Convolutional Neural Network," International Journal of Fuzzy Logic and Intelligent Systems, vol. 17(1), pp. 26-34, Mar 2017.

[26] Flower Recognition. Retrieved from www.kaggle.com/alxmamaev/flowers-recognition.

[27] Folio Data Set. Rerieved from http://archive.ics.uci.edu/ml/datasets/Folio.

\section{BIOGRAPHIES OF AUTHORS}

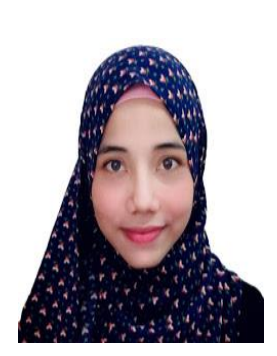

Nurul Fatihah Sahidan is currently taking Masters Degree in Computer Science (Web Technology) at the Faculty of Computer and Mathematical Sciences, Universiti Teknologi MARA, Shah Alam, Selangor, Malaysia. Her area of interests includes image processing and intelligent systems. 

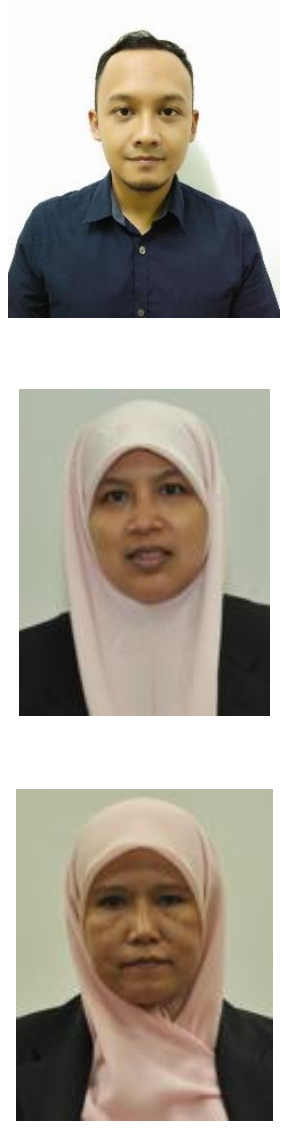

Ahmad Khairi Juha is currently taking Masters Degree in Computer Science (Web Technology) at the Faculty of Computer and Mathematical Sciences, Universiti Teknologi MARA, Shah Alam, Selangor, Malaysia. His area of interest includes image processing and intelligent systems.

Norasiah Mohammad is a Senior Lecturer at the Faculty of Computer and Mathematical Sciences, Universiti Teknologi MARA, Shah Alam, Selangor, Malaysia. She has been teaching courses related to Algorithms and Computer Science Education for more than ten years. Currently, she is actively involved in Interactive and Communication Technology (ICCT) research interest group where she has produced a few publications in the related area.

Zaidah Ibrahim is an Assoc. Prof. at the Faculty of Computer and Mathematical Sciences, Universiti Teknologi MARA, Shah Alam, Selangor, Malaysia. She has been teaching courses related to Artificial Intelligence for more than ten years. Currently, she is actively involved in Digital Image, Audio and Signal Technology (DIAST) research interest group where she has published a few publications in journals and present at conferences, nationally and internationally. 